\title{
Turkey Ovarian Tissue Transplantation: Effects of Surgical Technique on Graft Attachment and Immunological Status of the Grafts 6 Days Post- surgery
}

\section{George B Hall}

University of Guelph

Julie A. Long

United States Department of Agriculture

Leonardo Susta

University of Guelph

\section{Ben J. Wood}

The University of Queensland

Grégoy Yves Bédécarrats ( $\square$ gbedecar@uoguelph.ca )

University of Guelph Ontario Agricultural College https://orcid.org/0000-0001-6893-0624

\section{Research}

Keywords: Turkey, Ovary, Transplantation, Immunology, Rejection

Posted Date: July 28th, 2021

DOI: https://doi.org/10.21203/rs.3.rs-744107/v1

License: (c) (1) This work is licensed under a Creative Commons Attribution 4.0 International License.

Read Full License

Version of Record: A version of this preprint was published at Poultry Science on December 1st, 2021. See the published version at https://doi.org/10.1016/j.psj.2021.101648. 


\section{Abstract}

Background: Biobanked poultry ovaries can be revived via transplantation, into a recipient female, which upon maturity will produce donor-derived progeny. Previously, a large portion of these recipients also produced recipient-derived progeny, making them gonadal chimeras. These were potentially created when portions of the recipient's ovary were inadvertently left behind. Completely removing the recipient ovary would solve this problem, however, leaving a portion of the recipient's ovary may have inadvertently increased the transplant attachment rate by providing a damaged area for the transplant to attach too. To test this hypothesis in the turkey, we removed various portions (33\% to $100 \%$ ) of recipient ovarian tissue and determined the transplant attachment rate. Furthermore, the use of the abdominal air sac membrane as an additional anchoring point was tested. Finally, the immunological status of the grafts was evaluated by analyzing the presences of CD3 and MUM-1 (T and B cell markers), 6 days post-surgery.

Results: The overall attachment rate of transplants was $91 \%(32 / 35)$, while the average size of the transplants was $4.2 \pm 0.6 \mathrm{~mm}^{2}$. There was no difference $(P>0.05)$ in the attachment rates, or transplant size between groups with varying amounts of recipent tissue removed, or by using the abdominal air sac membrane as an anchor. However, all transplants were infiltrated by large numbers of $T$ and $B$ cells. This was shown by a high $(P \leq 0.001)$ percentage of CD3-positive immunostained cytoplasmic area (49.78 \pm $3.90 \%)$ in transplants compared to remnant recipient tissue $(0.30 \pm 0.10 \%)$, as well as a high $(P \leq 0.001)$ percentage of MUM-1-positive immunostained nuclear area $(9.85 \pm 1.95 \%)$ in transplants over remnant recipient tissues $(0.39 \pm 0.12 \%)$.

Conclusions: This study showed that neither the portion of the recipient ovary left behind nor the use of the abdominal air sac membrane affected the rate of attachment or the amount of donor tissue that attached. Thus, we recommend removing the entire recipient ovary to prevent gonadal chimeras. The high levels of lymphocytes within the grafts indicate possible tissue rejection, which could be overcome via immunosuppression with or without histocompatibility matching between donors and recipients.

\section{Background}

Historically within the poultry industry, government agencies, and universities, maintaining poultry breeds generation to generation has been the preferred preservation technique to conserve desirable genotypes and phenotypes. However, this is a costly practice, and even if the breed or line is commercially viable or relevant, the current trend is to discontinue the maintenance of live populations, when financial concerns arise. This has resulted in a loss of genetic diversity, and the elimination of valuable phenotypes and genotypes $[1,2]$. Thus, a more cost-effective model for preserving poultry breeds, needs to be established.

Biobanking of ovarian tissue and semen is a viable alternative to maintaining living populations and is estimated to be sixteen times more cost-effective than the current model [3]. Poultry semen cryopreservation techniques are quite advanced and have been researched extensively [4]. In contrast, cryopreservation and transplantation of poultry ovarian tissue is documented in only a handful of studies 
[5-7]. Presently, the limitation remains the low transplantation success rates, even when fresh tissue is used, which has reduced the widespread adoption of ovarian biobanking. Therefore, our focus has been to determine why fresh transplants have a low survival rate, and attempt to improve upon it [8]. To date, success has been defined as the production of donor-derived progeny. However, in most studies a majority of hens also produced recipient-derived progeny making them gonadal chimeras. As recipientderived progeny are a waste of financial resources, reducing their occurrence by eliminating gonadal chimeras has been previously attempted through removal of the entire recipient ovary either by using surgical forceps [9] or via cauterization [7]. The sterilization of recipients with busulfan before ovary removal and transplantation was also reported [10]. In chickens, the removal of the entire ovary alone, either with forceps or cauterization, still resulted in 6 out of $7(86 \%)$ hens, being gonadal chimeras $[7,9$, $10]$. When hens were sterilized with busulfan prior to the removal of the entire recipient's ovary, gonadal chimeras still developed. Although, a higher ratio of donor to recipient-derived progeny was achieved compared to birds that just had their ovaries removed [10]. In quail, removal of the entire ovary with forceps still resulted in 15 out of $27(56 \%)$ of the hens being gonadal chimeras $[5,6,11,12]$.

Since primordial germ cell migration occurs only during embryonic development in poultry, it is unlikely that the donor ovarian tissue was repopulated by recipient primordial germ cells, but rather, the recipient's ovary was not entirely removed leaving donor germ cells in situ [7]. Therefore, improving upon current techniques of removal, might lower the number of gonadal chimeras generated. However, we hypothesize that the remnant recipient tissue may have improved the attachment rate of the donor tissue by providing a traumatized area for the transplant to anchor to and for reanastomosis to occur. Because surgical anastomosis of major blood vessels is not possible for this procedure, successful transplantation relies on natural angiogenesis. Thus, removing all the recipient's tissue before transplantation might inadvertently decrease the number of transplants that successfully attach, therefore outweighing the benefits of reducing gonadal chimeras.

Therefore, our aim was to use the domestic turkey as a model to determine whether remnant recipient tissue is required for successful transplantation of ovarian tissue in the domestic turkey. Furthermore, it has also been suggested that laying the abdominal air sac membrane over the transplant might improve the attachment rate [9], which was also tested here. For analysis, appearance and cellular composition of the transplanted ovarian tissue was analysed to determine the immunological status 6 days posttransplantation.

\section{Methods}

\section{Animals and tissue collection}

Donors, recipients, surgical controls and unoperated female poults from a broad breasted white female parent-stock line were supplied by Hybrid Turkeys (Kitchener, Ontario Canada) at 1-day posthatch (dph). All animals were housed in the University of Guelph's Animal Care Facility, and all protocols were approved by the University of Guelph's Animal Care Committee (Animal Utilization Protocol \# 3921). 
Donor poults were given food and water ad libitum and housed in groups on wood shavings. Donor poults were euthanized via manual cervical dislocation at $8 \pm 1 \mathrm{dph}$. After euthanasia, the donor poult's ovary was removed and dissected into 2' $2 \mathrm{~mm}$ pieces and held in holding media (PBS with $20 \%$ Fetal Bovine Serum) at room temperature for no longer than 20 mins before being transplanted. The optimal age of donor ovarian tissue was chosen based on a previous study, with this age resulting in better germ cell and follicle morphology post-surgery [8]. Recipients and surgical controls were given only water and were kept in groups without shavings prior to surgery at 1 or $2 \mathrm{dph}$. Fasting ensured that the digestive track did not obscure the view of the ovary during surgery. Since poults still possess and rely upon the remnant yolk sac for nutrition at that age, fasting is not an issue. After surgery, recipients and surgical controls were immediately offered food and water ad libitum and were housed in the same manner as donors. An unoperated group served as controls and were treated the same as the recipient and surgical controls except they did not undergo surgery. Recipients, surgical controls and unoperated poults were all euthanized via manual cervical dislocation at 7 or $8 \mathrm{dph}, 6$ days post-surgery in the case of the recipients and surgical controls.

\section{Experimental groups}

Four surgical procedures were compared, and all involved allotransplants of fresh tissue placed orthotopically. In the first three groups, either $33 \%, 66 \%$ or $100 \%$ of the left ovary was removed before receiving the donor ovarian tissue ( $n=8 /$ group). In these groups, the dorsal portion of the left abdominal air sac membrane was fully opened, thus preventing it from covering or serving as an anchor for the transplants. In the fourth group, in addition to $100 \%$ ovary removal, the dorsal portion of the left abdominal air sac membrane was left partially intact over the transplants ( $n=6$ poults). Surgical controls had varying portions of the ovary removed but did not receive transplants ( $n=2 /$ group). Lastly, unoperated poults $(n=8)$ served as an additional control group.

\section{Transplantation of ovarian tissue}

Orthotopic transplantation of ovarian tissue between breeds has been reported in chicken, quail, and turkeys [9,11,13], while inter-species transplantation has also been reported for ducks [14]. Based on these previous studies, a modified procedure was used here. Firstly, recipient poults 1 to $2 \mathrm{dph}$ were moved to an intensive care unit (ICU; TLC-40, Brinsea Products Ltd., Florida, USA), set at $37.5^{\circ} \mathrm{C}$, to maintain body temperature before surgery. The poults were then weighed and given an intramuscular injection of butorphanol ( $2 \mathrm{mg} / \mathrm{kg}$ bodyweight [BW]) and returned to the ICU for 10 mins. An intramuscular injection of xylazine ( $2 \mathrm{mg} / \mathrm{kg} \mathrm{BW}$ ) was then administered. The poult was then placed in a supine position on a silicone pad on top of a heating plate $(15 \times 10 \mathrm{~cm}$, World Precision Instruments, Florida, USA), which was connected to an ATC2000 small animal temperature controller (World Precision Instruments) fitted with a mouse rectal probe (RET-3, World Precision Instruments) which was inserted into the cloaca of the bird. This equipment monitored poult temperature and adjusted the heating plate as required. A rodent 
facemask, connected to a Bain circuit, was then placed over the beak, with the oxygen flow rate set at 0.8 $\mathrm{L} / \mathrm{min}$. For induction, the isoflurane vapor was set at $1.5 \%$, and the anesthesia was maintained with the setting of the vaporiser ranging between 1 and $4 \%$. The respiration and heart rate, and rectal temperature were monitored and recorded manually. Before and during surgery, the pedal reflex response of pinching the skin between the toes was used to assess the depth of anesthesia. Once the poult was fully anesthetized, feathers on the left side of the abdomen were shaved with a small electric shaver (Fig. 1A). The skin was then cleaned with Germi-stat gel (Cardinal Health Canada, Ontario, Canada) followed by $70 \%$ ethanol. A $2 \mathrm{~cm}$ incision across the chick's abdomen (Fig. 1B) just below the ribs was then made using micro scissors (Vannas $8 \mathrm{~cm}$ with $5 \mathrm{~mm}$ blade, World Precision Instruments, Florida, USA). First, only the skin, rectus, and oblique abdominal muscles were cut, then, the peritoneal membrane was opened exposing the abdominal viscera (Fig. 1C). The yolk sac, which was located caudally within the abdominal cavity, was then gently pulled out to prevent rupturing (Fig. 1D). The connection between the yolk sac and intestine was ligated and then cut to remove the yolk sac. The abdominal viscera were then gently pushed down into the cavity the yolk sac previously occupied, allowing access to the ovary. At this point, a pair of front-lens-mounted PrismPro 8.0x surgical loupes (EVK 800, Surgitel, Michigan, USA) with a high intensity mini-LED mounted headlight were used to provide sufficient illumination and magnification. The ovary was reached by first pushing back the left hepatic lobe and gizzard, to expose the ventral aspect of the abdominal air sac (Fig. 1F). The air sac was opened via blunt dissection using micro forceps, exposing the dorsal portion of the membrane overlaying the ovary (Fig. 1G). For surgery groups 1 to 3 , this part of the abdominal air sac was opened via blunt dissection again to expose the ovary (Fig. 11). For surgery group 4, the dorsal part of the air sac was only partially opened below the ovary, keeping the portion overlaying the ovary intact. Varying portions of the ovary were then removed using micro forceps (Dumont $90^{\circ}$ bend, World Precision Instruments, Florida, USA). Poults in group 1 had $33 \%$ of the left ovary removed, along its right side, whereas group 2 had $66 \%$ of their left ovary removed along its right and left side (Fig. 2A-B), and groups 3 and 4 had all their left ovary removed. In all groups two pieces of donor tissue were positioned orthotopically (Fig. 1L). For group 4, the transplants were covered using the abdominal air sac membrane. The abdominal viscera were then gently repositioned over the transplants, and the abdominal muscles and skin were sutured closed (4-0 Monocryl PC-5 19 mm 3/8c Multipass, Ethicon, New Jersey, USA). A simple interrupted pattern was used with sutures spaced $\sim 2 \mathrm{~mm}$ apart. At the end of surgery, poults were given an intramuscular injection of ceftiofur hydrochloride ( $5 \mathrm{mg} /$ bird, EXCENEL ${ }^{\circledR}$ RTU), and a neck tag for identification purposes. The poults were then moved back into the intensive care unit, to recover from the anesthesia. Once recovered, poults were given meloxicam via crop gavage $\left(0.5 \mathrm{mg} /\right.$ bird, Metacam ${ }^{\circledR}$ Oral Suspension) for 3 days post-surgery to mitigate pain. Poults were weighed daily to confirm that they were eating and growing.

\section{Transplant processing, staining, and imaging}

Upon euthanasia of the recipients, surgical controls, and unoperated birds, the abdomen was opened and photographed using a dissecting microscope equipped with a camera (Leica EZ4 W; Optic-Tech Scientific Inc, Ontario, Canada). The recipient's ovary and surrounding tissue were then removed and fixed in Bouin 
solution for $1 \mathrm{hr}$ at RT, followed by an additional $23 \mathrm{hr}$ at $4{ }^{\circ} \mathrm{C}$ [15]. After fixation, the ovary was washed with PBS and transferred to a tissue cassette before being stored in $70 \%$ ethanol at $4{ }^{\circ} \mathrm{C}$ until processing. The ovary was then dehydrated and cleared with xylene and embedded in paraffin blocks as previously described for whole ovaries [15]. Transplants and recipient ovarian tissue were positioned in the paraffin blocks so that sectioning occurred parallel to the sagittal plane of the ovary. Ovaries were sectioned at a thickness of $5 \mu \mathrm{m}$ using a Finesse ME microtome (ThermoShandon, Cheshire, UK). Every $8^{\text {th }}$ section was collected through the transplants and recipient ovarian tissue, with five sections collected per slide and approximately 10 slides collected per ovary. Slides were stained with hematoxylin and eosin (H\&E) to allow examination of the entire specimen and ensured that small amounts of recipient tissue or transplants were not overlooked. During sectioning, two additional slides were created, each with five randomly collected sections. These two slides were used for immunohistochemistry analyses. The H\&E sections were imaged using a Leica DM 5000B light microscope (Leica, Wetzlar, Germany) equipped with a B-Series LED light source (ScopeLED, Richmond, CA, USA) for sequential red/green/blue imaging. Images were captured using a Hamamatsu Orca-Flash 4 camera (Hamamatsu Photonics, Hamamatsu City, Japan).

\section{Immunohistochemistry for CD3 and MUM-1.}

Immunohistochemistry for CD3 and MUM-1 antigens was performed at the University of Guelph Animal Health Laboratory (AHL), an American Association of Veterinary Laboratory Diagnosticians (AAVLD) accredited laboratory, using an automated staining instrument (Dako autostainer, Dako / Agilent, Ontario, Canada). Following manual deparaffinization and rehydration, the sections were treated with $3 \%$ hydrogen peroxide to quench endogenous peroxidase activity. For CD3, heat-induced epitope retrieval (HIER) was carried out using citrate buffer $(\mathrm{pH} 6)$ and a decloaking chamber (BioCare Medical, Concord, $\mathrm{CA}$, USA). For MUM-1, HIER was performed using an EDTA buffer $(\mathrm{pH} 9)$ and a similar pressure cooker device (PT Link, Dako). Primary antibodies against CD3 (rabbit polyclonal, Dako) and MUM-1 (mouse monoclonal, clone MUM1p, Dako) were diluted 1:100 and 1:50, respectively, and incubated at room temperature (RT) for 30 mins. Slides were then incubated with a dual anti-mouse / anti-rabbit anti-lgG horseradish peroxidase-linked polymer (EnVision for CD3, EnVision FLEX for MUM-1, Dako) for 30 mins at RT, and reactions were visualized using Nova Red chromogen (Vector Laboratories, Burlington, ON, Canada). Slides were counterstained with hematoxylin.

Tissue known to have high levels of T cells (thymus), and B cells (bursa of Fabricius) were used as positive controls for CD3 and MUM-1 IHC, respectively. These tissues were collected, fixed, and sectioned in the same way as for the transplants, although only a couple sections were collected, as needed. For negative controls, non-immune rabbit serum was substituted for anti-CD3 polyclonal antisera, and antibody diluent was substituted for MUM-1 antibody. Sections were imaged using the same microscope and camera setup as for H\&E slides. 


\section{Identification, size, and pathological analyses of recipient and transplanted ovarian tissues}

Remnant recipient ovarian tissue and transplants were first identified by comparing the surgical control birds to those that received donor ovarian tissue, using the gross dissecting microscope images. The size, shape, position, and color of the surgical control ovaries, remnant recipient tissue and transplants were all taken into consideration during the identification process. For birds that received donor tissue, ovarian tissue which was inconsistent with the ones from the surgical control birds were identified as possible transplants. Ovarian tissue which was consistent with the ones from the surgical control birds was classified as being remnant recipient ovarian tissue. Histological analysis of the potential grafts and remnant recipient tissue, using the H\&E slides, was carried out to confirm their identity. To be confirmed as remnant ovarian tissue, the tissue required attachment via the ovary ligament to the abdominal cavity. With the medulla possessing normal lacunar channels, and a well-defined medulla-cortex border, with the cortex having a mixture of prefollicular germ cells, and primordial follicles [15]. Conversely, transplants were classified as tissue with or without prefollicular germ cells and primordial follicles, that also lacked attachment via the ovarian ligament. Transplants could also be surrounded by fibrous connective tissue, around the entire or a portion of the transplant (sign of transplantation). The surgical control ovary, remnant recipient tissue and transplants were then measured (diameter and height), using the dissecting microscope gross images. The measurements were then used to calculate the area occupied by surgical control ovaries, remnant recipient tissue or transplants.

\section{Percent of positive CD3 and MUM-1 area}

The amount of $T$ and $B$ cells infiltrating the grafts was determined to evaluate the immunological status of the transplants. $T$ and $B$ cell infiltration was determined by the amount of cytoplasmic and nuclear immunostained area for CD3 and MUM-1, respectively. This was performed using a computer assisted image analysis tool in ImageJ (Open-source image processing software, 2.0.0-rc-69/1.52p). For each IHC stain, one image from each category (remnant recipient tissue, and transplant) was analyzed per bird. The image was captured at 40x magnification resulting in an area of approximately $105,625 \mu \mathrm{m}^{2}$ being analysed. For both IHC tests, the image colors were adjusted via the input levels using photoshop CC 2019 (Version 20, Adobe, California, USA). This was done to achieve a white background, changes were kept consistent with the two different IHC stains, thus improving the contrast between background and the stain. The color channel feature (color deconvolution) was used to separate the blue (Hematoxylin) and brown (Nova Red chromogen) color for each image. For the brown channel, the threshold feature was used to determine the percentage of immunostained (brown) area on each image, as expressed either by CD-3-positive cytoplasm or MUM-1-positive nuclear area, on a particular section. The total nuclear (blue) and cytoplasm (white) area on each image was determined on the blue channel. For CD3, the percentage of immunostained area was divided by the percentage of total cytoplasm to provide a percent of 
immunostained cytoplasmic area. For MUM-1, the percent of immunostained nuclei area was divided by the percent of total nuclei area, corresponding to the percent of immunostained nuclei area.

\section{Statistical analysis}

For bird survival, recipient ovary presences, and size values were combined between the birds that received transplants and their respective surgical controls, for analysis. Statistical analyses were performed using SPSS 25.0 for Mac (SPSS Inc., Chicago, IL). For each experimental group, the average ovarian size (surgical control, remnant recipient tissue or transplant) and percentage of CD3- or MUM-1immunostained area were presented as means \pm standard error of the mean (SEM). Normality and equal variance of the data were evaluated by residual plots and Levene's tests, respectively. A one-way ANOVA followed by a post-hoc (Tukey) test was used to analyze the effect of surgery group. Significance was set at $(P £ 0.05)$. For poult survival and presence of ovarian tissue or transplants within the surgical controls or recipients, the data was presented in count form with percentages. The data was analyzed via a generalized linear model (binary logistic), to determine if the surgery group affected the survival rate or presence of remnant ovarian tissue or transplants. After an effect $(P £ 0.05)$ was determined, a pair-wise comparison was run to determine the varying groups.

\section{Results}

\section{Surgery survival rates}

Of the 45 surgeries performed, 37 poults (82\%) survived with 1 poult further euthanized post-surgery resulting in an overall study survival rate of $80 \%$ (Table 1 ). The cause of death during surgery was attributed to exsanguination after a major blood vessel was inadvertently damaged, while the poult euthanized two days after surgery was not feeding or gaining weight sufficiently. The highest survival rate $(91 \%)$ was seen in the two groups which had $33 \%$ and $100 \%$ of the recipient ovary removed.

Compared to these two groups, the survival rate $(60 \%)$ was lower $(P \leq 0.01)$ in the group which had $100 \%$ of the recipient ovary removed and had the abdominal air sac membrane only partially opened. On a technical note, leaving the abdominal air sac membrane intact obstructed the view of the ovary, and blood vessels below, which increased the difficulty level of the procedure.

\section{Table 1}

Surgery survival, along with transplant, and recipient tissue presences and size 


\begin{tabular}{|c|c|c|c|c|c|c|c|c|}
\hline \multirow[t]{3}{*}{ Group } & Surgical & Surgeries & \multicolumn{2}{|c|}{ Birds } & Recipent & Recipent & Ovarian & Ovarian \\
\hline & Procedure & Performed & \multicolumn{2}{|c|}{ Surviving } & Ovary & Ovary & Transplant & Transplant \\
\hline & & & \multicolumn{2}{|c|}{$\begin{array}{l}\text { (\% } \\
\text { survived) }\end{array}$} & Present & $\begin{array}{l}\text { Size } \\
\mathrm{mm}^{2}\end{array}$ & $\begin{array}{l}\text { Present } \\
(\%)\end{array}$ & Size $\mathrm{mm}^{2}$ \\
\hline UO & $\begin{array}{l}0 \% \\
\text { removed }\end{array}$ & - & - & & - & $\begin{array}{l}16.6 \pm \\
0.8^{a}\end{array}$ & - & - \\
\hline 1 & $\begin{array}{l}33 \% \\
\text { removed } \\
+ \\
\text { transplant }\end{array}$ & 8 & 8 & $\begin{array}{l}(91) \\
\mathrm{a}\end{array}$ & $100 \%^{a}$ & $\begin{array}{l}9.0 \pm \\
1.1^{\mathrm{b}}\end{array}$ & $\begin{array}{c}7 \\
(88)^{a}\end{array}$ & $3.4 \pm 1.2^{a}$ \\
\hline $1 \mathrm{SC}$ & $\begin{array}{l}33 \% \\
\text { removed }\end{array}$ & 3 & 2 & & & & - & - \\
\hline 2 & $\begin{array}{l}66 \% \\
\text { removed } \\
+ \\
\text { transplant }\end{array}$ & 11 & 8 & $\begin{array}{l}(77) \\
a b\end{array}$ & $90 \%^{a}$ & $\begin{array}{l}4.1 \pm \\
1.0^{\mathrm{c}}\end{array}$ & $\begin{array}{c}7 \\
(88)^{a}\end{array}$ & $4.8 \pm 1.2^{a}$ \\
\hline $2 \mathrm{SC}$ & $\begin{array}{l}66 \% \\
\text { removed }\end{array}$ & 2 & 2 & & & & - & - \\
\hline 3 & $\begin{array}{l}100 \% \\
\text { removed } \\
+ \\
\text { transplant }\end{array}$ & 9 & 8 & $\begin{array}{l}(91) \\
a\end{array}$ & $10 \% \mathrm{~b}$ & $\begin{array}{l}0.0 \pm \\
0.0^{d}\end{array}$ & $\begin{array}{c}8 \\
(100)^{a}\end{array}$ & $5.3 \pm 1.5^{a}$ \\
\hline $3 S C$ & $\begin{array}{l}100 \% \\
\text { removed }\end{array}$ & 2 & 2 & & & & - & - \\
\hline 4 & $\begin{array}{l}100 \% \\
\text { removed } \\
+ \\
\text { transplant, }\end{array}$ & 10 & 6 & $\begin{array}{l}(60) \\
b\end{array}$ & $33 \% \mathrm{~b}$ & $\begin{array}{c}0.7 \pm \\
0.5^{\mathrm{cd}}\end{array}$ & $\begin{array}{c}5 \\
(83)^{a}\end{array}$ & $4.0 \pm 1.4^{a}$ \\
\hline
\end{tabular}


Total

$U O=$ unoperated $S C=$ surgical control

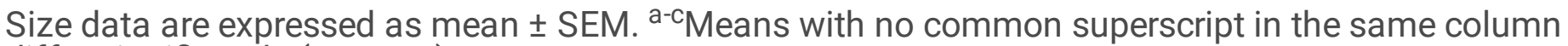
differ significantly $(P<0.05)$

\section{Differentiation of recipient ovarian tissue and transplants}

To identify grafts and remnant recipient ovarian tissue, comparisons were made between the surgical controls and the birds that received transplants (Figure 3). From a gross anatomical perspective, the remnant recipient ovary appeared more translucent, seen by the clear appearance of the adrenal gland, and major blood vessels positioned dorsally (Fig. 3B) compared to the opaquer transplants (Fig. 3B). Transplants also had a white to cream colour in contrast to the pale pink color of the recipient ovary (Fig. 3D). These gross descriptions were consistent for all transplants and remnant recipient tissue in this study. After remnant tissue and transplants were identified, further histological analysis was performed to confirm the origin of the tissue. The remnant recipient tissue appeared histologically normal with the presences of a distinct cortex and medulla containing lacunar channels (Fig. 4A). Within the cortex, prefollicular germ cells and immature granulosa cells were present (Fig. 4B). In contrast, the transplants appeared as coalescing inflammatory foci (Fig. 4A), rich in blood vessels and composed mainly of what appeared to be lymphocytes, with scattered macrophages and heterophils (Fig. 4C). The presence of blood vessels indicated that there was blood flow to these inflammatory foci. Scattered throughout this inflammation, were primordial follicles (Fig. 3D) and prefollicular germ cells (Fig. 3E). Fibrosis was also seen around some of the transplants (Fig. 3F).

\section{T and $B$ lymphocyte infiltration}

In addition to the visual comparison, immunohistochemistry for CD3 (T cell marker) and MUM-1 (B cell maker) demonstrated that the remnant recipient tissue had few lymphocytes present within. This was in stark contrast to the transplants which were highly immunostained for these T and B cell markers (Fig. 5). A substantial increase in the percentage of CD3 immunostained cytoplasmic area $(P \leq 0.001)$ was observed between the remnant recipient tissue $(0.30 \pm 0.10 \%)$ and the transplants $(49.78 \pm 3.90 \%)$. This was also the case $(P=0.033)$ for the percentage of MUM-1 immunostained nuclei area with $0.39 \pm 0.12 \%$ and $9.85 \pm 1.95 \%$ for the remnant recipient tissue and transplants, respectively. The overwhelming 
presence of $T$ and $B$ cells was consistent with inflammation or graft rejection, although the specific function of these lymphocytes was not determined in the present study.

\section{Recipient ovarian tissue and transplant presences and size}

As shown in Table 1, partial removal of the recipient ovary (either $33 \%$ or $66 \%$ ) increased $(P \leq 0.01)$ the presence of recipient tissue 6 days post-surgery, compared to the groups which had all the recipient's ovary removed. Within the two groups that had $100 \%$ removal, the group that did not use the abdominal air sac membrane to cover the transplants, had 1 recipient (10\%) still possessing its own ovarian tissue, although the piece was very small. Whereas the group which used the partially intact abdominal air sac membrane to cover the transplants, had 2 recipients (33\%) still possessing their own ovarian tissue. In this group, the remaining recipient tissue appeared to be in the most cranial portion, which was located furthest from the point at which the abdominal air sac membrane was partially opened.

As expected, there was a significant $(P \leq 0.001)$ drop in ovarian size between the unoperated group (16.6 $\left.\pm 0.8 \mathrm{~mm}^{2}\right)$ and the group which had $33 \%$ of the recipient ovary removed $\left(9.3 \pm 1.1 \mathrm{~mm}^{2}\right)$. There was a further decrease $(P=0.001)$ in remnant ovarian size between the $33 \%$ removal group and the group which had $66 \%$ removed $\left(4.2 \pm 1.2 \mathrm{~mm}^{2}\right)$.

Overall, the attachment rate (presences of grafts) was $90 \%$ (27 out of 30 ), varying between 83 and $100 \%$ across individual surgery groups, however, there no difference was seen $(P=0.556)$ between groups. The average size of the transplants was $4.4 \pm 0.6 \mathrm{~mm}^{2}$, meaning only 1 out of the 2 transplanted pieces successfully attached, or both attached and then reduced in size. There was also no statistical difference $(P=0.699)$ in transplant size between surgery groups.

\section{Discussion}

The successful transplantation of fresh turkey ovarian tissue is a vital first step in establishing a working protocol for biobanking. Ovarian transplantation in poultry breeds is a challenging procedure, and although success in other species has been reported $[9,11,14]$, a detailed and comprehensive account of the procedure is absent from the literature making it difficult to replicate. Here, we first present a step-bystep approach on the procedure, guided by visual references to ensure ease of replication.

For ovarian transplantation work, the first crucial step is to ensure survival of the poults during surgery, as the most common cause of death is exsanguination due to accidental damage to the left illaca communis vein, a major blood vessel positioned dorsal to the ovary. The use of surgical loupes, as previously reported [13], allowed for greater precision and accuracy in avoiding this major blood vessel. With the use of loupes and improved surgical ability, we were able to increase the survival rate compared to the above-mentioned paper. In the present study, differences in survival rates were observed between surgery groups. The highest survival rates were seen when $33 \%$ and $100 \%$ of the recipient ovary was removed, showing that survival rate is not influenced by the amount of recipient tissue removed. 
Conversely, the lowest poult survival rate was seen in the group which used the abdominal air sac membrane to cover the transplants. With the abdominal air sac membrane left intact, the view of the ovary was obstructed making it difficult to avoid blood vessel damage, and thus resulting in higher mortality. Unfortunately, a majority of studies in other avian species do not report surgery survival rates, making comparison with chicken, quail, and duck studies difficult $[6,7,9,12,14]$. Furthermore, out of the studies which did report survival rates, no differentiation was made between deaths on the operating table or deaths during the recovery period $[5,10,11]$.

As suturing the ovarian transplant in place is not feasible due to its fragility and size, the second crucial step is optimizing the condition for successful attachment. To improve the attachment rate, previous groups have used the abdominal air sac membrane to cover the transplants [7,9], making it challenging to see the recipient ovary, which might have led to recipient ovarian tissue being accidently left behind. This remnant tissue may have improved the attachment rate, by providing a damaged area to graft too, although this would also increase the chances of creating gonadal chimeras. To determine the relevance of these factors, we compared removing varying amounts of recipient ovarian tissue, and placing transplants under the abdominal air sac membrane. Overall, the effective removal of varying portions of recipient ovarian tissue was validated post-surgery by evaluating the presence and size of remnant recipient tissue. However, raising the birds to sexual maturity to produce offspring would be the only way to confirm without doubt the full removal of the recipient ovary. From our results the amount of recipient tissue remaining and the use of the abdominal air sac membrane to anchor the transplants had no effect on the presence nor the size of the transplants 6 days post-surgery. This suggests that the remnant recipient tissue is not a necessary attachment point for the transplants, and the abdominal air sac membrane does not aid in anchoring the transplant. Therefore, to ensure the least amount of recipientderived progeny are produced, it seems most advantageous to remove the entire recipient ovary, and not to use the abdominal air sac membrane as an anchor. In fact, trying to keep the abdominal air sac membrane intact while removing the recipient's ovary resulted in the largest proportion of recipient tissue being mistakenly left behind, which may explain why so many gonadal chimeras have been reported, to date $[5-7,9,10-12]$.

Interestingly, in a chorioallantoic membrane (in-ovo) culture system, we reported attachment rates of $100 \%$ when $7 \mathrm{dph}$ ovarian tissue was used as grafts [8], and based on previous studies in quail $[11,16]$ we hypothesized that results from in-ovo culturing might be transferable to live birds. The overall graft attachment rate in the present study (90\%) suggests that indeed in-ovo culture system might be able to predict the attachment rate when the tissue is transplanted into a living bird.

Beyond poult survival, the amount of recipient ovarian tissue remaining, and surgical technique, the last crucial step investigated was to determine the immunological status of the transplants. In mammalian whole organ transplantation, failure is usually caused by immunological rejection, when the recipient's immune system recognizes the organ as foreign. However, tissue damage brought on by ischemia and reperfusion injury may also activate the inflammatory response [17]. Both rejection and inflammation can cause the tissue to become infiltrated by leukocytes, in particular T and B cells, in the case of rejection 
$[18,19]$. As expected, low levels of $T$ and $B$ cells were detected in the remnant recipient tissue, however, transplants were highly infiltrated with lymphocytes. Out of the 2 types of cells identified by immunohistochemistry, it appears most lymphocytes present within the transplants were $T$ cells, which is expected in the case of acute cellular rejection [20]. It should be noted that the B cell marker (MUM-1) used here was developed from plasma cells, and so some naïve B cells might not have been detected.

Although cellular functional analysis was not performed, morphology was consistent with graft rejection, although inflammation cannot be ruled out until an autotransplant is compared to an allotransplant. As 6 days post-surgery was the only time point evaluated in our study, we cannot rule out that the lymphocyte infiltration occurred at an earlier stage. This is earlier than the 14 days graft rejection reported in skin transplantation between unsuppressed chickens [21], however, the timing here is in line with the average acute rejection seen (3 to 10 days post-surgery) in mammals $[22,23]$.

Immunosuppressants were not used in this study as in previous work, they were demonstrated not to be a requirement for success. In fact, when gonads were transplanted between chickens of the same breed, and recipient were unsuppressed, transplants were successful $71 \%$ of the time [24]. Conversely, when transplantation was performed across different breeds in chickens and quails, $25 \%$ and $17 \%$ of the hens produced donor-derived progeny, respectively $[9,11]$. The fact that immunosuppressants were not required in these previous studies was explained by the theory of acquired tolerance $[7,25]$. Acquired tolerance has been suggested to be inducible during a short window before or just after hatch in chickens [26]; however, more recent findings suggest this window occurs earlier during embryonic development [27] with immunocompetency reached at around the 14th day of embryonic development [28]. Thus, acquired tolerance may not be possible to induce at the time of hatch. In this study, transplant morphology and the high levels of $\mathrm{T}$ and $\mathrm{B}$ cell infiltration strongly suggest immune rejection even though both donors and recipients were from the same breed. Based on this high level of infiltration, it is doubtful that these transplants would be viable, in stark contrast to what was previously reported in chickens, which had ovarian tissue transplanted between the same breed [24]. Without further timepoints, we cannot rule out that the infiltration may subside, allowing the grafts to return to a functional state. Although incompatibility has been reported between different breeds in poultry [24], to the best of our knowledge, it has not been reported within the same breed.

\section{Conclusions}

Ovarian transplantation in turkey poults is a delicate and complex surgery, and for effective partial or full removal of the ovary, the use of surgical loupes is recommended. The amount of remaining recipient ovarian tissue did not affect the rate of transplant attachment, nor did covering the transplant with the abdominal air sac membrane. Therefore, removing the entire recipient ovary before transplantation, and not using the abdominal air sac membrane is recommended to decrease the likelihood of creating gonadal chimeras, and recipient-derived progeny. This technique resulted in $91 \%$ attachment rate, well above reported values in any poultry species $[7,11,14,13]$. However, based on the high level of CD3 and MUM-1 positive area within the transplants, it appears an immune rejection mediated by $T$ and $B$ 
lymphocytes is taking place by 6 days post-transplantation. It is therefore suggested that recipients be treated with an immunosuppressant following transplantation, or donors and recipients be immunologically matched before transplantation. Although to date, the type and dose of immunosuppressant to be used still needs to be optimized for turkeys.

\section{Declarations}

\section{Acknowledgements}

We would like to first thank the Central Animal Facility staff at the University of Guelph (ON) for taking care of the research animals. We also acknowledge the technical support from Jodi Morrison (Ontario Veterinary College, Guelph, ON) and Dr. Michaela Strueder-Kypke (Molecular and Cellular Imaging Facility, Guelph, ON), for assisting with histology and microscopy, respectively. Lastly, were appreciate the hard work and support from Dr. Josepha Delay and Susan Lapos (Histotechnology Laboratory, Animal Health Laboratory, University of Guelph).

\section{Authors' contributions}

GH performed the experimental procedure, analyzed the data and wrote the manuscript. JL provided technical and financial assistance through the USDA support aggreements and contributed to the experimental design. LS assisted with the IHC experimental design, analyses and interpretation. BW procurred animals and participated in the design of the surgical procedure. GB provided funding through the USDA support aggreements and NSERC Discovery program, oversaw the execution of the experiment, and particpated in the interpretation of the data. All authors contributed to the editing of the manuscript.

\section{Funding}

This research was supported by United States Department of Agriculture via two support agreements (588042-7-068-F and 58-8042-9-070-F), and by the Natural Sciences and Engineering Rsearch Council of Canada (Discovery Grant Program). Additionally, poults were provided in-kind by Hybrid Turkeys, Canada.

\section{Availability of data and materials}

The datasets used and analyzed during this study are available from the corresponding author upon request.

\section{Ethics approval and consent to participate}


All experimental and surgical procedures followed the approved Animal Utilization Protocol (AUP \#3921) granted by the Institutional Animal Care Committee according to the principles of the Canadian Council on Animal Care.

\section{Consent for publication}

Not applicable.

\section{Competing interests}

The authors declare that they have no competing interests

\section{References}

1. Muir WM, Wong GK, Zhang Y, Wang J, Groenen MA, Crooijmans RP, Megens HJ, Zhang H, Okimoto R, Vereijken A, Jungerius A. Genome-wide assessment of worldwide chicken SNP genetic diversity indicates significant absence of rare alleles in commercial breeds. P Natl Acad Sci. 2008;105:173127.

2. Long JA, Blackburn H, Martin A, Taylor RL Jr, Silversides F, Youngs CR. Protecting food animal gene pools for future generations. Issue Paper-Council for Agricultural Science Technology. 2019;65:24.

3. Silversides FG, Purdy PH, Blackburn HD. Comparative costs of programmes to conserve chicken genetic variation based on maintaining living populations or storing cryopreserved material. Brit Poultry Sci. 2012;53:599-607.

4. Sarkar PK. Motility, viability and fertilizing ability of avian sperm stored under in vitro conditions. Rev Agr Sci. 2020;8:15-27.

5. Liu J, Song Y, Cheng KM, Silversides FG. Production of donor-derived offspring from cryopreserved ovarian tissue in Japanese quail (Coturnix japonica). Biol Reprod. 2010;83:15-9.

6. Liu J, Cheng KM, Silversides FG. A model for cryobanking female germplasm in Japanese quail (Coturnix japonica). Poultry Sci. 2013;92:2772-5.

7. Liptoi K, Buda K, Rohn E, Drobnyak A, Meleg EE, Palinkas-Bodzsar N, Vegi B, Barna J. Improvement of the application of gonadal tissue allotransplantation in the in vitro conservation of chicken genetic lines. Anim Reprod Sci. 2020;213:106280.

8. Hall GB, Long JA, Wood BJ, Bedecarrats GY. In ovo culturing of turkey (Meleagris gallopavo) ovarian tissue to assess graft viability and maturation of prefollicular germ cells and follicles. Poultry Sci. 2020;99:7109-21.

9. Song Y, Silversides FG. Offspring produced from orthotopic transplantation of chicken ovaries. Poultry Sci. 2007;86:107-11. 
10. Song Y, Silversides FG. Long-term production of donor-derived offspring from chicken ovarian transplants. Poultry Sci. 2008;87:1818-22.

11. Song Y, Silversides FG. Transplantation of ovaries in Japanese quail (Coturnix japonica). Anim Reprod Sci. 2008;105:430-7.

12. Liu J, Cheng KM, Silversides FG. Recovery of fertility from adult ovarian tissue transplanted into week-old Japanese quail chicks. Reprod Fert Develop. 2015;27:281-4.

13. Hall G. Vitrification of day old turkey testes and ovaries, and subsequent transplantation and folliculogenesis growth rates and patterns in chickens https://harvest.usask.ca/handle/10388/ETD2015-10-2272 (2015) Accessed 1 July 2021.

14. Song Y, Cheng KM, Robertson MC, Silversides FG. Production of donor-derived offspring after ovarian transplantation between Muscovy (Cairina moschata) and Pekin (Anas platyrhynchos) ducks. Poultry Sci. 2012;91:197-200.

15. Hall GB, Long JA, Wood BJ, Bedecarrats GY. Germ cell dynamics during nest breakdown and formation of the primordial follicle pool in the domestic turkey (Meleagris gallopavo). Poultry Sci. 2020;99:2746-56.

16. Liu J, Cheng KM, Silversides FG. Novel needle-in-straw vitrification can effectively preserve the follicle morphology, viability, and vascularization of ovarian tissue in Japanese quail (Coturnix japonica). Anim Reprod Sci. 2012;134:197-202.

17. Lentsch AB, Kato A, Yoshidome H, McMasters KM, Edwards MJ. Inflammatory mechanisms and therapeutic strategies for warm hepatic ischemia/reperfusion injury. Hepatology. 2000;32:169-73.

18. Ingulli E. Mechanism of cellular rejection in transplantation. Pediatr Nephrol. 2010;25:61-74.

19. Sakai Y, Kobayashi M. Lymphocyte 'homing' and chronic inflammation. Pathol int. 2015;65:344-54.

20. Benzimra M, Calligaro GL, Glanville AR. Acute rejection. J Thorac Dis. 2017;9:5440.

21. Wick G, Müller PU, Schwarz S. Effect of cyclosporin A on spontaneous autoimmune thyroiditis of Obese strain (OS) chickens. Eur J Immunol. 1982;12:877-81.

22. Wood KJ, Goto R. Mechanisms of rejection: current perspectives. Transplantation. 2012;93:1-10.

23. Owen JA, Punt J, Stranford SA, Jones PP, Owen JA, Punt J, Stranford SA. Effector responses: cell-and antibody-mediated immunity. In: Freeman WH, editor. Kuby Immunology 7th ed. New York: 2013. p. $427-44$.

24. Liptoi K, Horvath G, Gal J, Varadi E, Barna J. Preliminary results of the application of gonadal tissue transfer in various chicken breeds in the poultry gene conservation. Anim Reprod Sci. 2013;141:869.

25. Hedger MP. Immunologically privileged environments. In: Halberstadt C, Emerich D, editors. Cellular transplantation: from laboratory to clinic. California: Elsevier; 2007. pp. 567-90.

26. Billingham RE, Brent L, Medawar PB. Quantitative studies on tissue transplantation immunity. III. Actively acquired tolerance. Philos T Roy Soc B. 1956;65:357-414. 
27. Șereș M, Igna C, Cioca D, Schuszler L. Bone marrow cells versus mature lymphocytes and monocytes-capacity of immunotolerance induction in COBB 500 hybrids. Bulletin of University of Agricultural Sciences and Veterinary Medicine Cluj-Napoca. Veterinary Medicine. 2008;65:222-7.

28. Tizard IR. Innate immunity: the recognition of invaders. In: Tizard IR, editor. Veterinary immunology: an introduction. 8th ed. Philadelphia: Saunders; 2008. pp. 11-20.

\section{Figures}






\section{Figure 1}

Ovarian transplantation in the domestic turkey. a The poult was first anaesthetised and placed in the supine position, the abdominal feathers were removed by an electric shaver, $b$ an incision was made through the abdominal skin and muscle wall to expose the internal viscera c. The liver (arrow), and gizzard (arrowhead), are clearly visible. $d$ The yolk sac was removed from the abdomen, then the connection between the yolk sac and small intestine (arrow) was ligated and cut to allow for the yolk sac 
to be detached. e The entry point above the ovary is located between the gizzard and liver, once pushed aside, the left abdominal air sac (arrow) and left posterior thoracic air sac (arrowhead) can be viewed. The most ventral portion of the left abdominal air sac membrane is then torn open, leaving the left posterior thoracic air sac (arrowhead) intact $\mathrm{f}$. $\mathrm{g}$ The dorsal portion of the abdominal air sac can then be grasped, and fully or partly torn open depending on the surgery group. h The ovary (arrow), and mesonephros kidney (arrowhead), can be seen clearly. i a small portion of the ovary can be gripped with a forceps and removed $\mathrm{j}$. $\mathrm{k}$ When the ovary is removed, the adrenal glands (arrow) can be viewed with the mesonephros kidney (arrowhead) still being present. I The transplants (arrows) are then laid orthotopically, after which the abdominal viscera are pushed back into place, and the opening is sutured closed. The poult depicted in these images was euthanized beforehand, live animals were not used in this figure. Scale bars (A-D) $1.0 \mathrm{~cm}$, (E-L) $2.0 \mathrm{~mm}$.

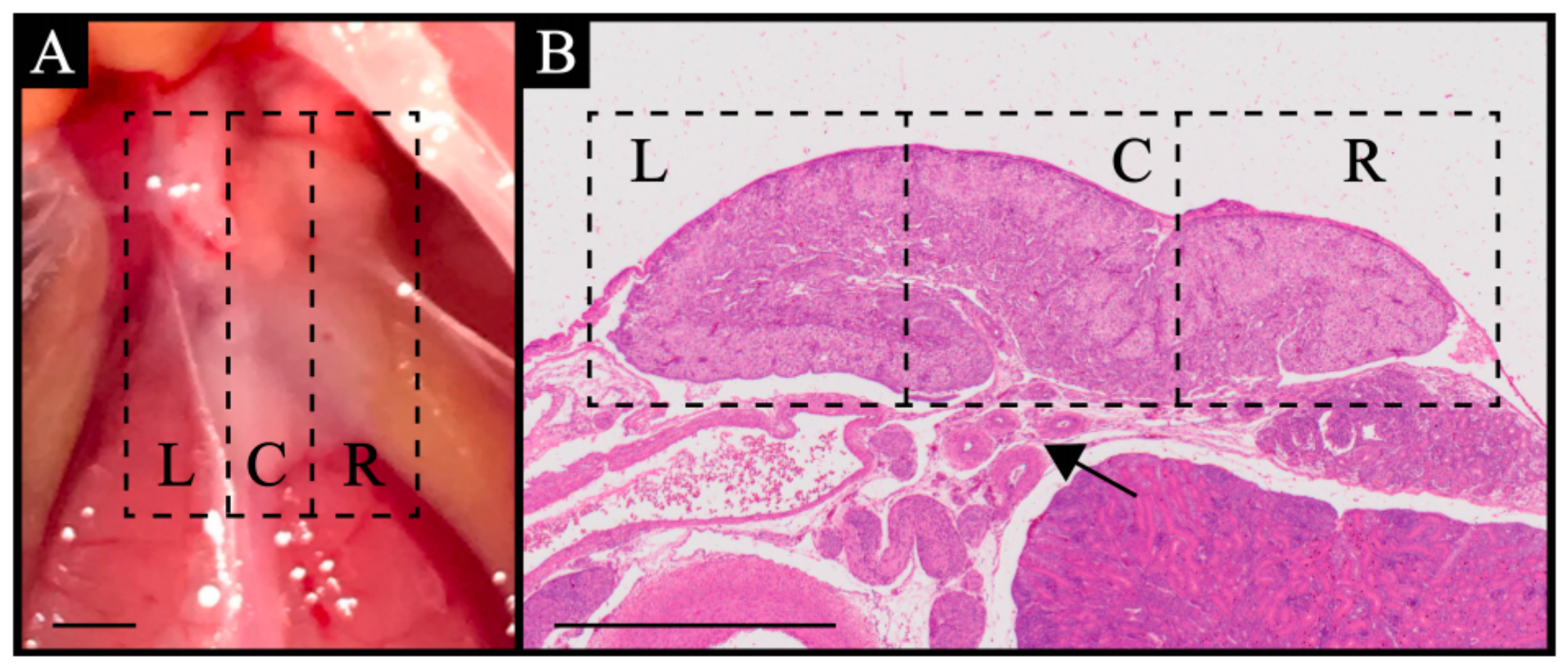

\section{Figure 2}

Representative day-old turkey ovary. a Gross anatomical perspective. b Histological perspective. The ovary ligament (arrow) is located in the centre portion c, allowing the left $(L)$ and right $(R)$ sides to be removed individually, without causing the remaining portions to detach. Scale bars $1 \mathrm{~mm}$. 


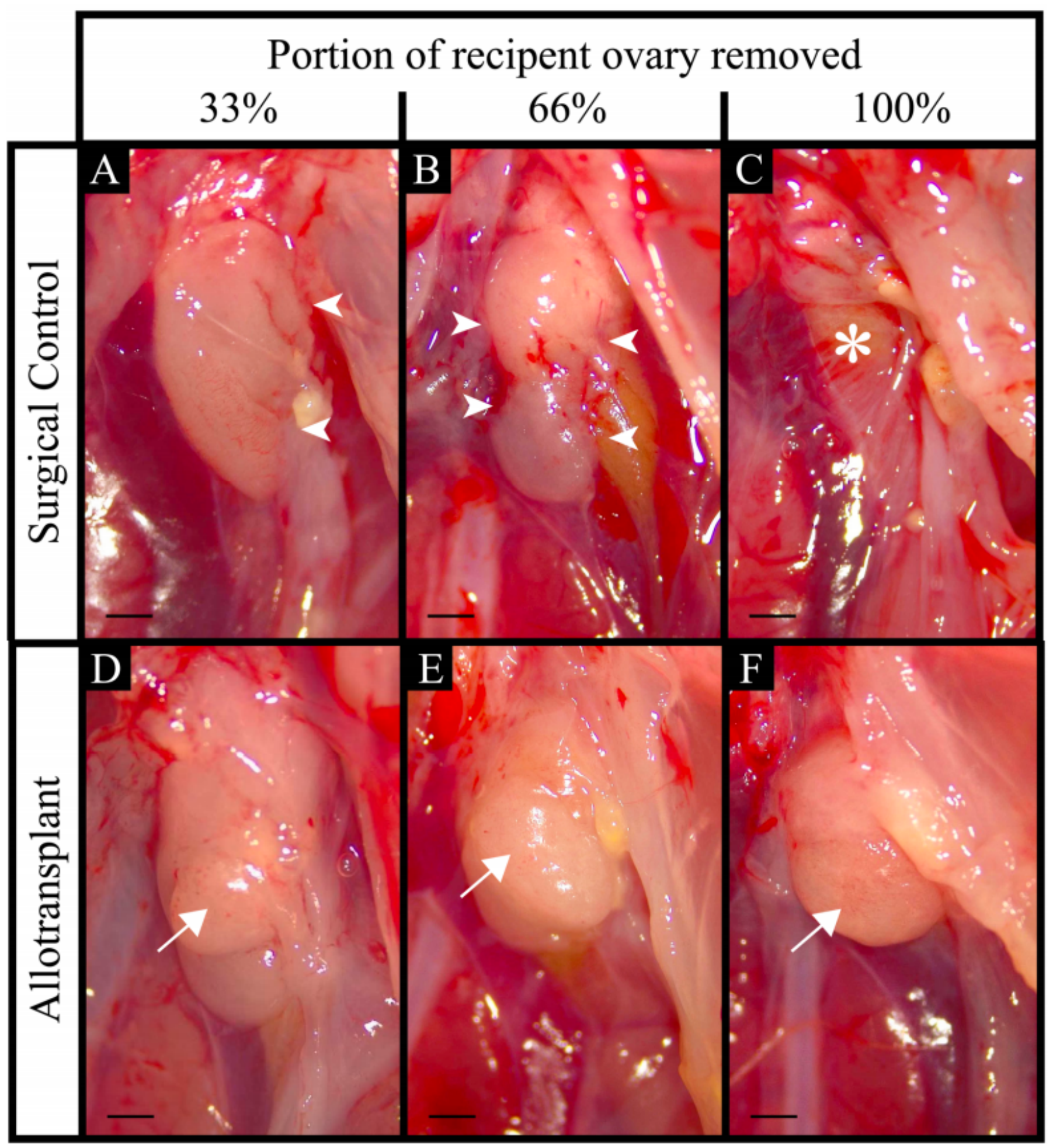

Figure 3

Gross anatomy of transplants and surgical controls 6 days post-surgery. $a$, b Surgical control ovaries showing clear signs of damage along the sides (arrow heads), where portions were removed. c Entire ovary removal, the asterisk designates prior location. $d$ - $f$ Identified transplants (arrows). Scale bars 1.0 $\mathrm{mm}$. 


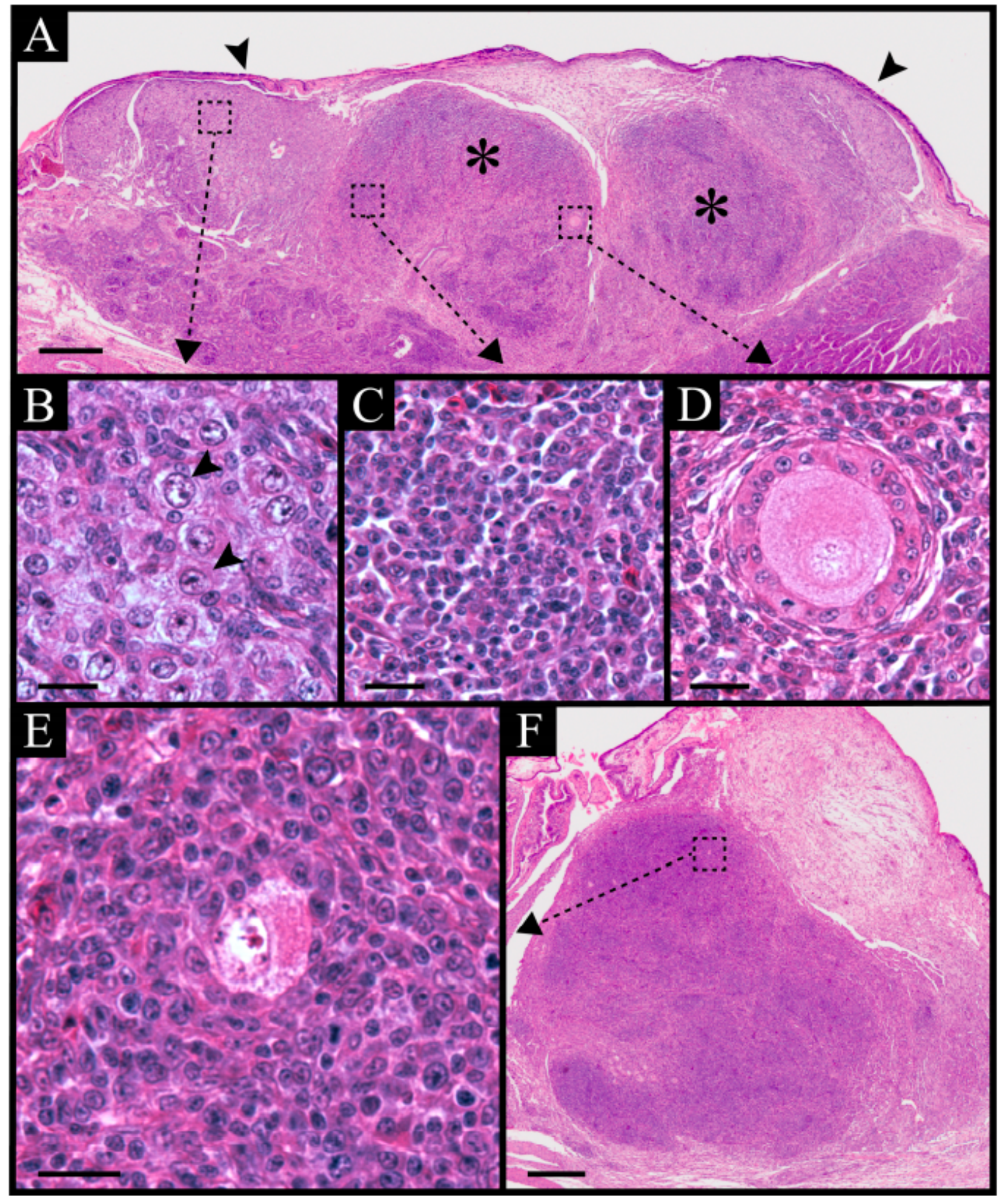

\section{Figure 4}

Histological images of transplants and remnant recipient tissue. a Cross section of two transplants (asterisk) with $66 \%$ of the recipient ovary still being present (arrowheads). The cortex and medulla of the recipient tissue can still be seen, whereas the transplants are heavily infiltrated by coalescing inflammatory foci (asterisks). b - d Correspond to higher magnifications of the dotted squares. b The remnant recipient ovary cortex appears normal with prefollicular germ cells (arrowheads), and immature 
granulosa cells situated around the prefollicular germ cells. c The inflammatory foci appear predominantly composed of lymphocytes (small cells, high nuclear/cytoplasm ratio, with a round nucleus, and dispersed chromatin with one prominent central nucleolus). $d$ Primary follicle found in the centre of a transplant. e Corresponds to higher magnifications of the dotted squares showing a prefollicular germ cell in the centre of a transplant surrounded by apparent lymphocytes. f Full cross section of the transplant in a $100 \%$ ovary removal recipient, many fibres connective cells can be seen in the top right-hand corner. Scale bars a, f $300 \mu \mathrm{m}$, b - e $20 \mu \mathrm{m}$.

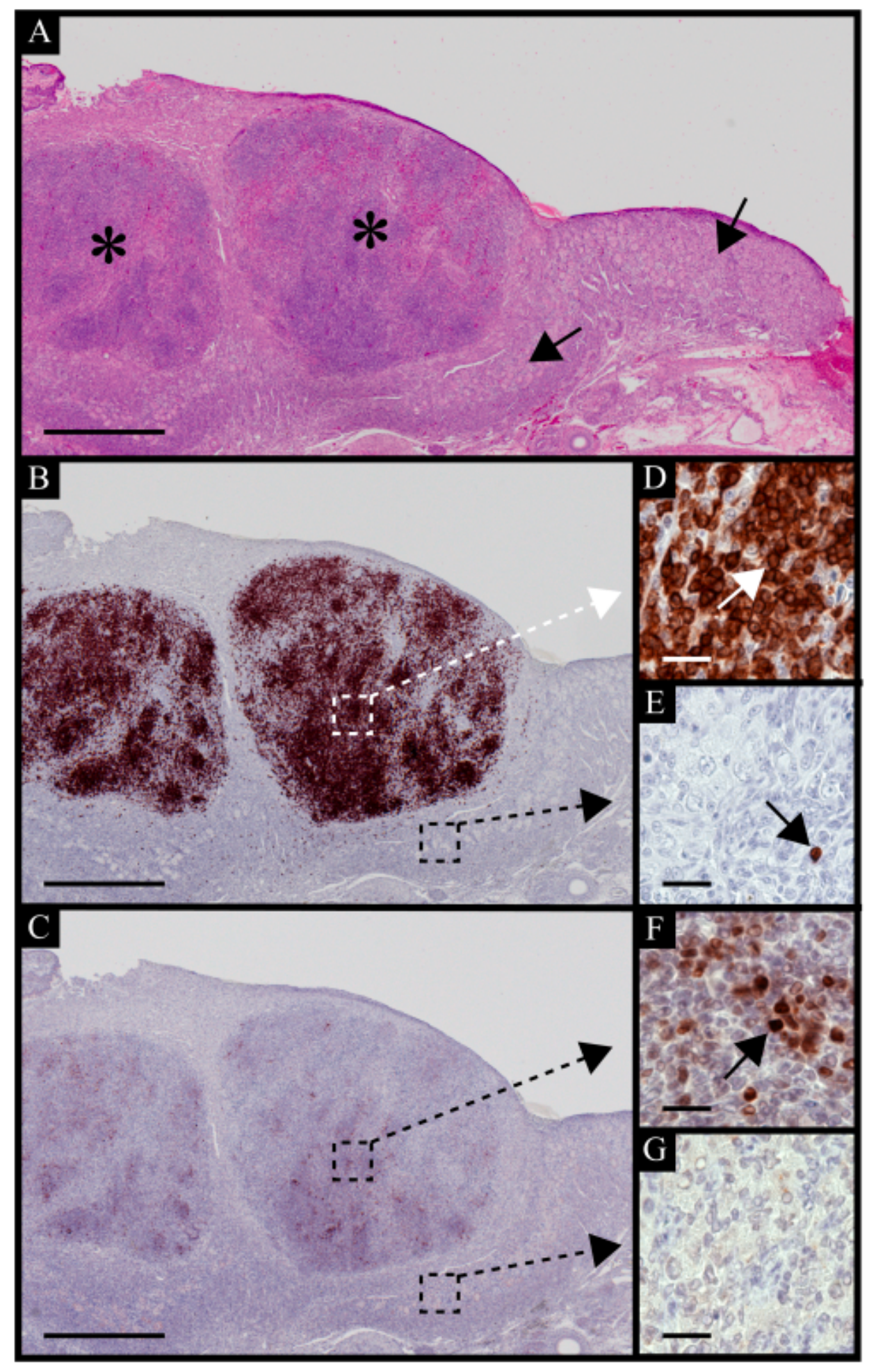




\section{Figure 5}

T and B cell infiltration of the allotransplants. a Shows two transplants (asterisk) above the remnant recipient ovary (arrows). b and c show the same segment after immunohistochemistry for CD3 and MUM1 respectively. The areas positive for CD3 and MUM-1 display as brown, whereas the counter stain is seen as blue. $d$ - $g$ Correspond to higher magnifications of the dotted squares. $d$ Shows most of the cells within the transplant are T lymphocytes (arrows) while e shows very few T lymphocytes within the remnant recipient tissue. $f$ shows $B$ lymphocytes (arrows) present in the transplant while $g$ highlights the minimal amount of $B$ cells within the remnant recipient tissue. Scale bars $(a-c, e, g, i) 500 \mu m,(d, f, h, j) 20 \mu m$. 